\title{
Enjeux et conséquences de la vannerie dans les aires protégées de Madagascar
}

\section{Sylvie Guillaud}

Cédric Vermeulen

Université de Liège

Faculté de Gembloux Agro-Bio Tech

Département BIOSE

Passage des déportés, 2

5030 Gembloux

Belgique

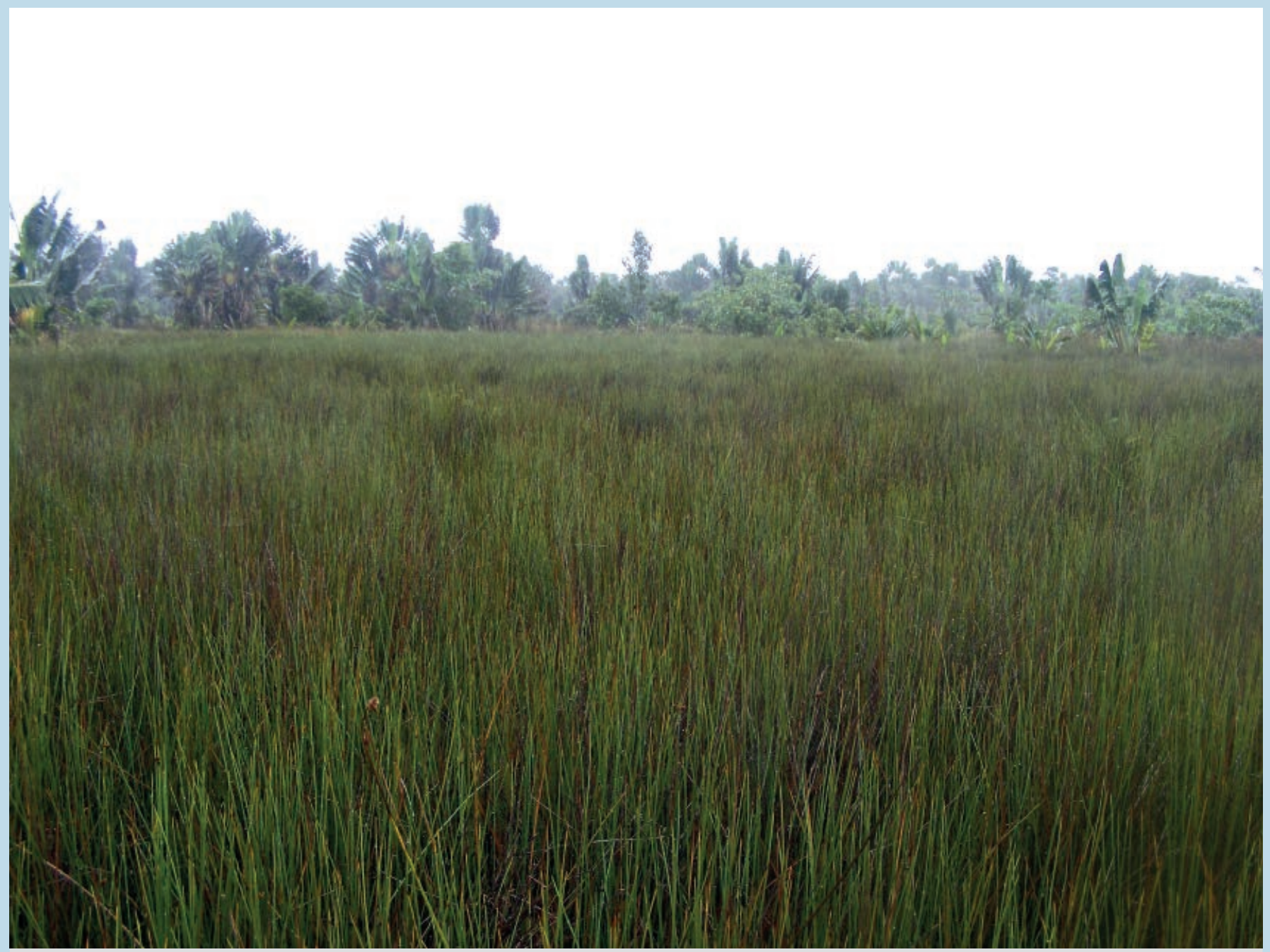

Photo 1.

Marais d'Amparahibe.

Photo S. Guillaud. 


\section{RÉSUMÉ}

\section{ENJEUX ET CONSÉQUENCES DE LA VANNERIE DANS LES AIRES PROTÉGÉES DE MADAGASCAR}

La Réserve spéciale de Manombo (Sud-Est de Madagascar) est un cas typique de zone de conflits entre conservation de l'environnement et développement rural. Elle contient un vestige de forêt littorale subissant des pressions constantes exercées par les populations riveraines qui, faute d'alternatives économiques, dépendent fortement de leur environnement. La vannerie représente une activité traditionnelle importante et les peuplements de Lepironia mucronata (mahampy), Cypéracée utilisée dans la vannerie, subissent une collecte excessive de tiges. Dans ce travail, trois peuplements de L. mucronata ont été étudiés afin d'évaluer l'impact de la vannerie sur cette ressource. Pour cela, des mesures de hauteur, de diamètre et de densité de tiges ont permis d'évaluer la productivité de chaque peuplement, et ainsi des différences ainsi significatives ont été constatées entre les trois marais. Parallèlement, une méthode indirecte d'estimation a été mise au point pour évaluer la pression annuelle sur les marais, exprimée en nombre de femmes venant se fournir en tiges. Outre confirmer la surexploitation de cette ressource par la vannerie, cette approche permet de déduire le nombre maximal de femmes pouvant se procurer des tiges sans menacer la régénération naturelle de $L$. mucronata. Enfin, des enquêtes socioéconomiques réalisées auprès des ménages et des commerçantes ont permis d'analyser la filière vannerie traditionnelle ainsi que les retombées économiques dans la région. Cela a permis de mettre en évidence le rôle primordial de cette activité, qui procure souvent les seuls revenus monétaires de nombreux ménages ruraux.

Mots-clés : Lepironia mucronata Rich., vannerie, filière, pressions anthropiques, ressources naturelles, produit forestier non ligneux (PFNL), Atsimo-Atsinanana, Madagascar.

\section{ABSTRACT}

\section{BASKETRY IN MADAGASCAR'S PROTECTED AREAS: ISSUES AND CONSEQUENCES}

The Manombo Special Reserve in south-east Madagascar is typical of zones where conflicts arise between environmental conservation and rural development. The vestigial coastal forest it contains is under constant pressure from communities in the vicinity, which, for lack of any economic alternative, are highly dependent on their immediate environment. Basketry is an important traditional activity and the stems of Lepironia mucronata (mahampy), a swamp grass of the Cyperacea family used in basketwork, are being harvested in excessive quantities. Three $L$. mucronata swamps were investigated for this study in order to assess the impact of basketry on the resource. Measurements of the height, diameter and density of the grass stems were used to assess the productivity of each area, and showed significant differences between the three swamps. In parallel, an indirect estimation method was developed to assess annual pressure on the swamps, expressed in terms of the number of women coming to harvest stems. This approach confirmed that the resource is being overexploited, but also enabled us to deduce the maximum number of people who can harvest stems without endangering the natural regeneration of $L$. mucronata. Finally, we analysed the traditional basketwork sector and its economic impacts in the region through socio-economic surveys among households and the women selling their basketwork. The results show the essential role of their activities, which are often the only source of monetary income for many rural households.

Keywords: Lepironia mucronata Rich., basketry, sector, human pressure, natural resources, non timber forest product (NTFP), Atsimo-Atsinanana, Madagascar.

\section{RESUMEN}

\section{RETOS Y CONSECUENCIAS DE LA CESTERÍA EN LAS ÁREAS PROTEGIDAS DE MADAGASCAR}

La Reserva Especial de Manombo (sudeste de Madagascar) es un caso típico de zona de conflictos entre conservación ambiental y desarrollo rural. Aquí se encuentra un vestigio de bosque litoral sometido a las constantes presiones ejercidas por las poblaciones colindantes que, ante la falta de alternativas económicas, dependen en gran medida de su entorno natural. La cestería supone una importante actividad tradicional y los rodales de Lepironia mucronata (mahampy en malgache), Cyperaceae empleada en cestería, sufren una recolección excesiva de sus tallos. En este trabajo se estudiaron tres rodales de L. mucronata para evaluar el impacto de la cestería en este recurso. Para ello, se realizaron mediciones de altura, diámetro y densidad de tallos que permitieron evaluar la productividad de cada rodal, pudiéndose observar diferencias significativas entre las tres zonas pantanosas. Simultáneamente, se puso a punto un método indirecto de estimación para evaluar la presión anual sobre los pantanos, expresada en el número de mujeres que vienen a recolectar tallos. Además de confirmar la sobreexplotación de este recurso por la cestería, este enfoque permite deducir el máximo número de mujeres que pueden recoger tallos sin amenazar la regeneración natural de $L$. mucronata. Por último, las encuestas socioeconómicas realizadas a hogares y comerciantes permitieron analizar el sector de la cestería tradicional y las repercusiones económicas en la región. Esto permitió poner de manifiesto el papel esencial de esta actividad que, a menudo, proporciona los únicos ingresos monetarios de muchos hogares rurales.

Palabras clave: Lepironia mucronata Rich., cestería, sector, presiones antrópicas, recursos naturales, productos forestales no madereros, Atsimo-Atsinanana, Madagascar. 


\section{Introduction}

Par sa biodiversité globale, son niveau d'endémisme et son taux de déforestation, Madagascar est un des plus grands hot spots mondiaux pour la conservation de la biodiversité (MYERS et al., 2000 ; GANZHORN et al., 2001 ; CONSIGLIO et al., 2006). Le long de la côte Est de l'île, une étroite bande de forêt se développant sur sable présente une diversité exceptionnelle avec plus de 1535 espèces végétales relevées. Elle est référencée comme forêt littorale (CADOTTE et al., 2002). À l'origine, cette forêt littorale formait une bande presque continue de $1600 \mathrm{~km}$ le long de la côte Est de Madagascar, couvrant environ 465100 ha (GREEN, SUSSMAN, 1990). Actuellement, il n'en resterait plus que 47900 ha, répartis en quelques fragments isolés, tous sujets à des pressions constantes de la part des populations riveraines. Parmi ces fragments, seuls 1,5\% sont inclus dans le réseau national d'aires protégées, dans la très dégradée Réserve spéciale de Manombo et dans le Parc national de Masoala (KREMEN et al., 1999).

Dans le cas de la Réserve spéciale de Manombo, les pressions exercées sur la forêt littorale trouvent leurs origines dans les besoins de base que les populations riveraines cherchent à satisfaire. Dans cette région, les sources de revenus sont rares et se répartissent en trois activités principales : la pêche, les cultures de rente et la vannerie (REZA et al., 2005). Les deux premières activités se concentrent uniquement dans certaines zones, pour des raisons géographiques (accès à la mer) et historiques (pratique des cultures de rente héritée du passé colonial). La vannerie, quant à elle, est pratiquée à une échelle beaucoup plus large et est souvent complémentaire aux deux activités précédentes. Lorsque ces dernières sont absentes, la vannerie devient la seule source de revenus. Cette situation place les populations concernées en situation de forte dépendance face à la disponibilité de la matière première : le mahampy, Lepironia mucronata, Cyperaceae non endémique se développant dans certains marais boisés et désormais surexploités par cette activité (REZA et al., 2005).

Le mahampy est une plante vivace qui se multiplie à partir de rhizomes et présente des tiges cylindriques appréciées pour la vannerie (RAZANAJATOVO, 2006 ; RANDRIATAFIKA et al., 2007). Selon IKUSIMA (1978), chaque rhizome porte en moyenne cinq tiges vivantes qui atteignent leur maturité après sept mois, une nouvelle tige émergeant de chaque rhizome en moyenne toutes les sept à huit semaines.

La présente étude s'est penchée sur l'exploitation des tiges de mahampy, considérée comme un des moteurs principaux de la pénétration dans l'aire protégée. Afin d'évaluer son importance sociale et économique, la filière vannerie a été étudiée à l'échelle de la région d'Atsimo-Atsinanana, depuis l'exploitation des tiges dans les marais jusqu'à la vente de vannerie dans les marchés urbains régionaux. Par ailleurs, le stade de développement, la productivité et l'état de dégradation des peuplements de L. mucronata ont été étudiés dans trois marais. En couplant cette étude de végétation aux paramètres de productivité de peuplements de $L$. mucronata issus de la recherche de IKUSIMA (1978) et au suivi de pressions des zones collectées, la recherche s'est concentrée pour confirmer la surexploitation de L. mucronata et évaluer les modalités d'exploitation durable des tiges pour la vannerie dans la zone d'étude.

\section{Matériels et méthodes}

\section{Sites d’étude}

L'étude a été menée sur trois marais situés à proximité ou à l'intérieur de deux forêts littorales protégées du district de Farafangana, entre les villes de Vangaindrano et de Farafangana, région d'Atsimo-Atsinanana (figure 1).

La première forêt se trouve dans la Réserve spéciale de Manombo. Elle est localisée à cheval entre quatre communes rurales: Ankarana, Manambotra, Labohazo et Mahabo/Mananivo. Elle est gérée par l'Association nationale pour la gestion des aires protégées (Angap). Située entre $47^{\circ} 38^{\prime}$ et $47^{\circ} 48^{\prime}$ de longitude Est et $22^{\circ} 58^{\prime}$ et $23^{\circ} 08^{\prime}$ de latitude Sud, cette réserve fut créée en décembre 1962 (ANGAP, 2003). Elle est constituée de deux parcelles. La parcelle 2 abrite la forêt littorale, qui s'étend sur 1055 ha et est caractérisée par la présence d'Intsia bijuga et d'Uapaca littoralis.

La seconde forêt est celle d’Agnalazaha, gérée par le Missouri Botanical Garden et située dans la commune rurale de Mahabo/Mananivo. D'une superficie d'environ 1565 ha, cette forêt est comprise entre $47^{\circ} 41^{\prime}$ et $47^{\circ} 45^{\prime}$ de longitude Est et $23^{\circ} 09^{\prime}$ et $23^{\circ} 14^{\prime}$ de latitude Sud.

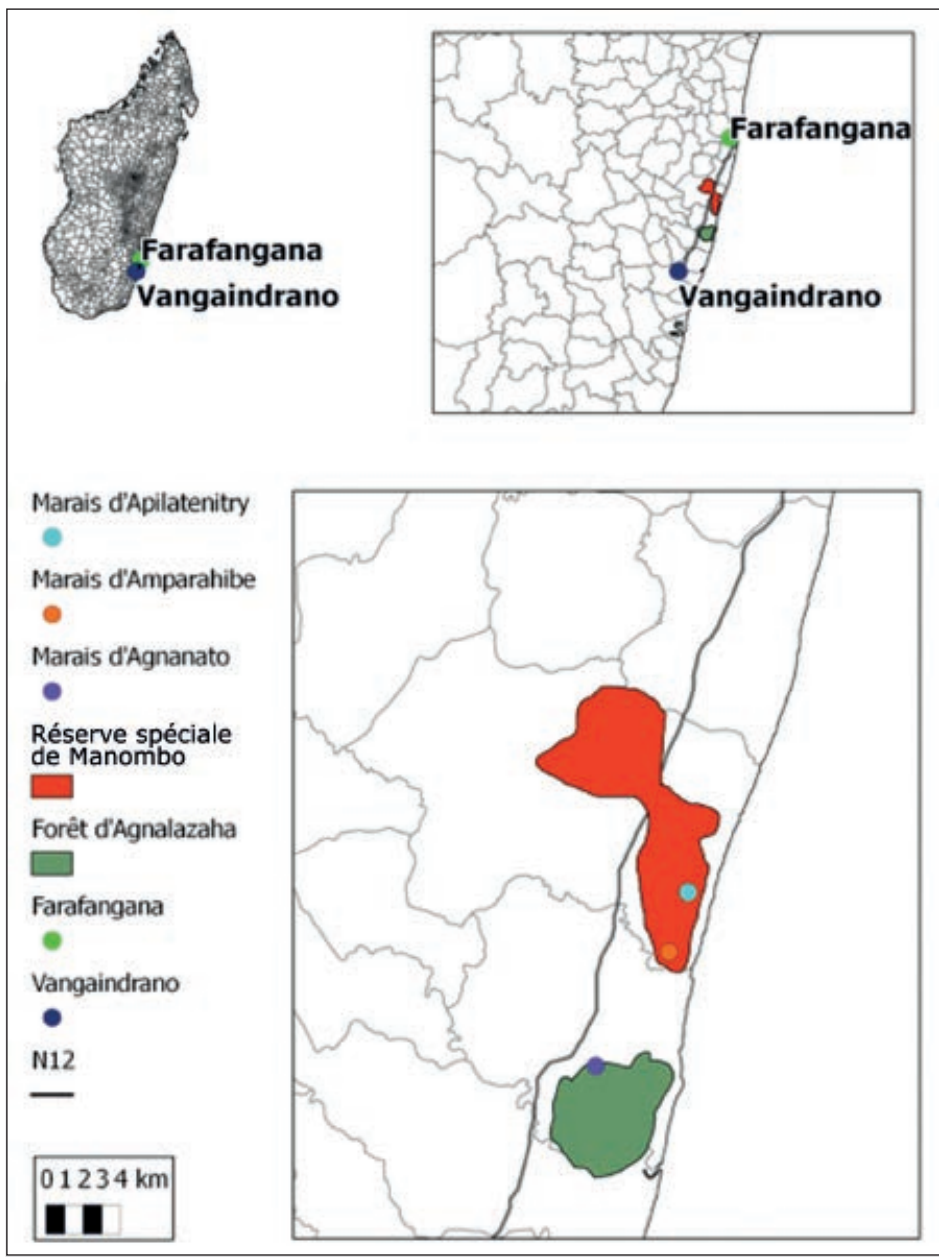

Figure 1

Localisation de la zone d'étude dans la région d'AtsimoAtsinanana, Sud-Est de Madagascar.

Source : S. Guillaud. 


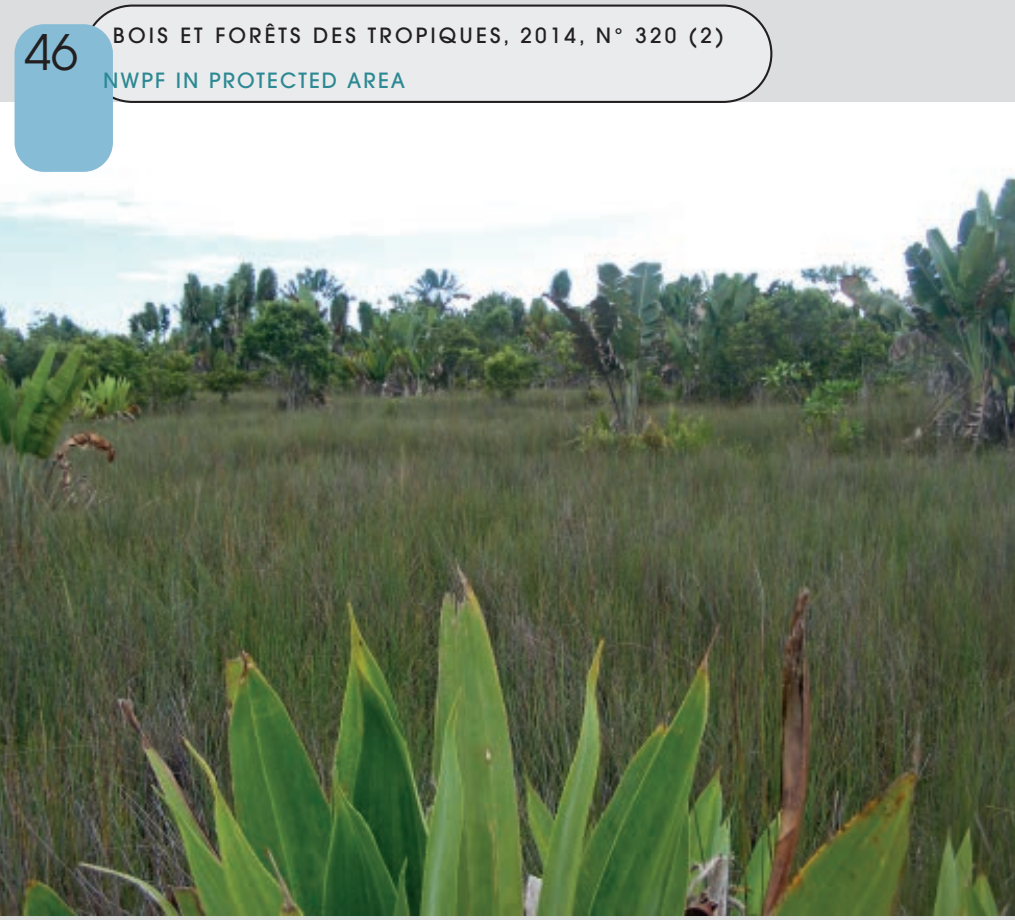

Photo 2.

Marais d'Apilatenitry.

Photo S. Guillaud.

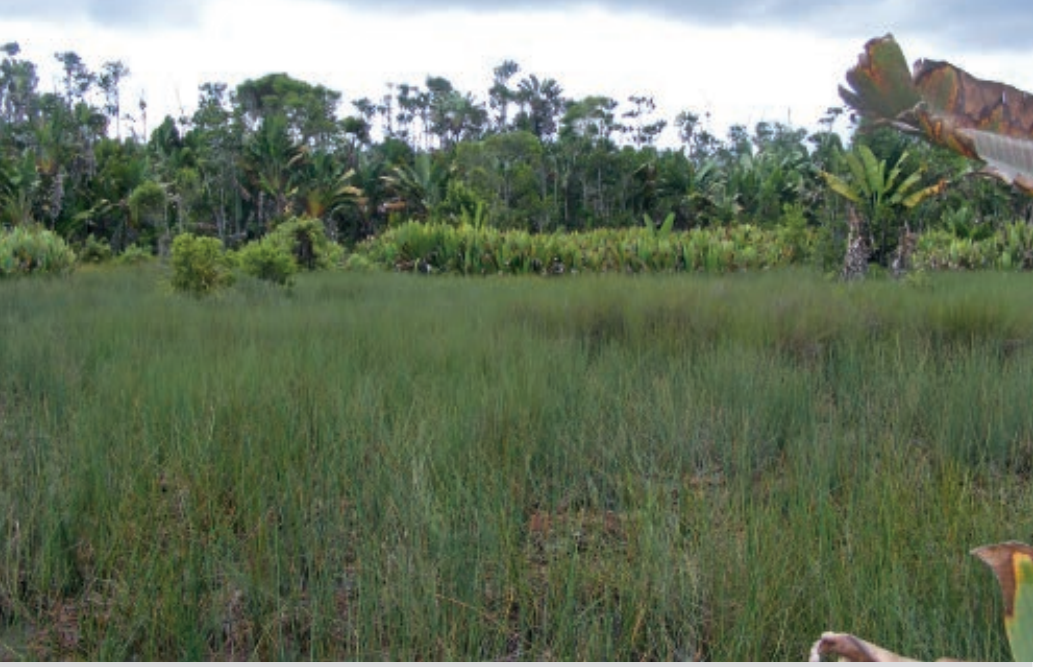

Photo 3.

Marais d'Agnanato.

Photo S. Guillaud.

Le choix des marais s'est appuyé sur les contacts des deux structures d'accueil avec les populations riveraines. Ces dernières vivent dans de petits villages souvent unilignagers et d'une même ethnie, regroupés administrativement en fokontany (unité qui comprend des hameaux, des villages, des secteurs ou des quartiers). Chaque marais est principalement exploité par les femmes d'un même fokontany, situé à proximité de celui-ci. La densité de la population de la zone est relativement faible, atteignant $69 \mathrm{hab} . / \mathrm{km}^{2}$ pour la commune de Mahabo/Mananivo (REZA et al., 2005) et $56 \mathrm{hab} . / \mathrm{km}^{2}$ pour la zone de Manombo (ANGAP, 2003).
Comme l'indique la figure 1 , deux des trois marais étudiés sont situés dans la Réserve spéciale de Manombo. Le premier marais est celui d'Amparahibe (photo 1), il se trouve à l'extrême Sud de cette réserve et est exploité par les femmes du fokontany de Karimbelo, appartenant toutes à l'ethnie Antesaka. Le deuxième marais est celui d'Apilatenitry (photo 2), situé au centre de l'aire protégée. Il est exploité par les femmes du fokontany de Takoandra, dont les populations appartiennent à l'ethnie Antevato.

Le dernier marais étudié est le marais d'Agnanato (photo 3) et se trouve à proximité de la forêt littorale d'Agnalazaha. Il est exploité principalement par les femmes des fokontany de Mahabo et de Vohimasy, de l'ethnie Antesaka.

\section{Relevés de végétation}

Toutes les données ci-dessous ont été récoltées sur des unités d'échantillonnage (UE) matérialisées sur le terrain à l'aide de quatre piquets et d'un ruban, formant ainsi un quadrat d'un mètre carré. Étant situés sur des marais tous largement exploités pour la vannerie, l'ensemble des quadrats ont, de ce fait, subi une certaine pression anthropique.

Ces trois marais possèdent une flore peu diversifiée, constituée principalement de L. mucronata et de Sphagnum $\mathrm{sp}$. Ces deux espèces présentent un taux de recouvrement important, au milieu duquel se développent quelques autres espèces, principalement des Poacées et des Cypéracées.

\section{Plan d'échantillonnage}

La conception des grilles d'échantillonnage a été mise au point après l'observation des trois sites d'étude. Chaque marais a été délimité à l'aide d'un appareil Gps Garmin $60 c s x$ en mode "track» et la trace obtenue a été téléchargée sur un ordinateur grâce au logiciel de gestion de récepteur de Gps Garbel-2.6. La trace fut ensuite utilisée sur Grille-3.2, un logiciel permettant de créer des grilles d'échantillonnage.

Afin de couvrir de manière uniforme chaque zone marécageuse, un échantillonnage systématique à deux dimensions a été choisi, avec un taux de sondage influencé par la variabilité de la végétation et par la taille du marais.

Une fois la dimension de la maille choisie, la grille a été téléchargée sur l'appareil Gps qui, par la fonction navigateur, a permis de localiser sur le terrain toutes les UE formant la maille.

\section{Stade de développement}

Le stade de développement des tiges de L. mucronata a été observé au sein de chaque UE et les tiges ont été réparties en trois catégories :

- jeune tige en développement sans inflorescence ;

- tige développée avec inflorescence ouverte ;

- phase de sénescence et mort.

Les tiges en floraison représentent le stade auquel le mahampy est le plus souvent collecté car il présente alors une hauteur et une grosseur suffisantes. 


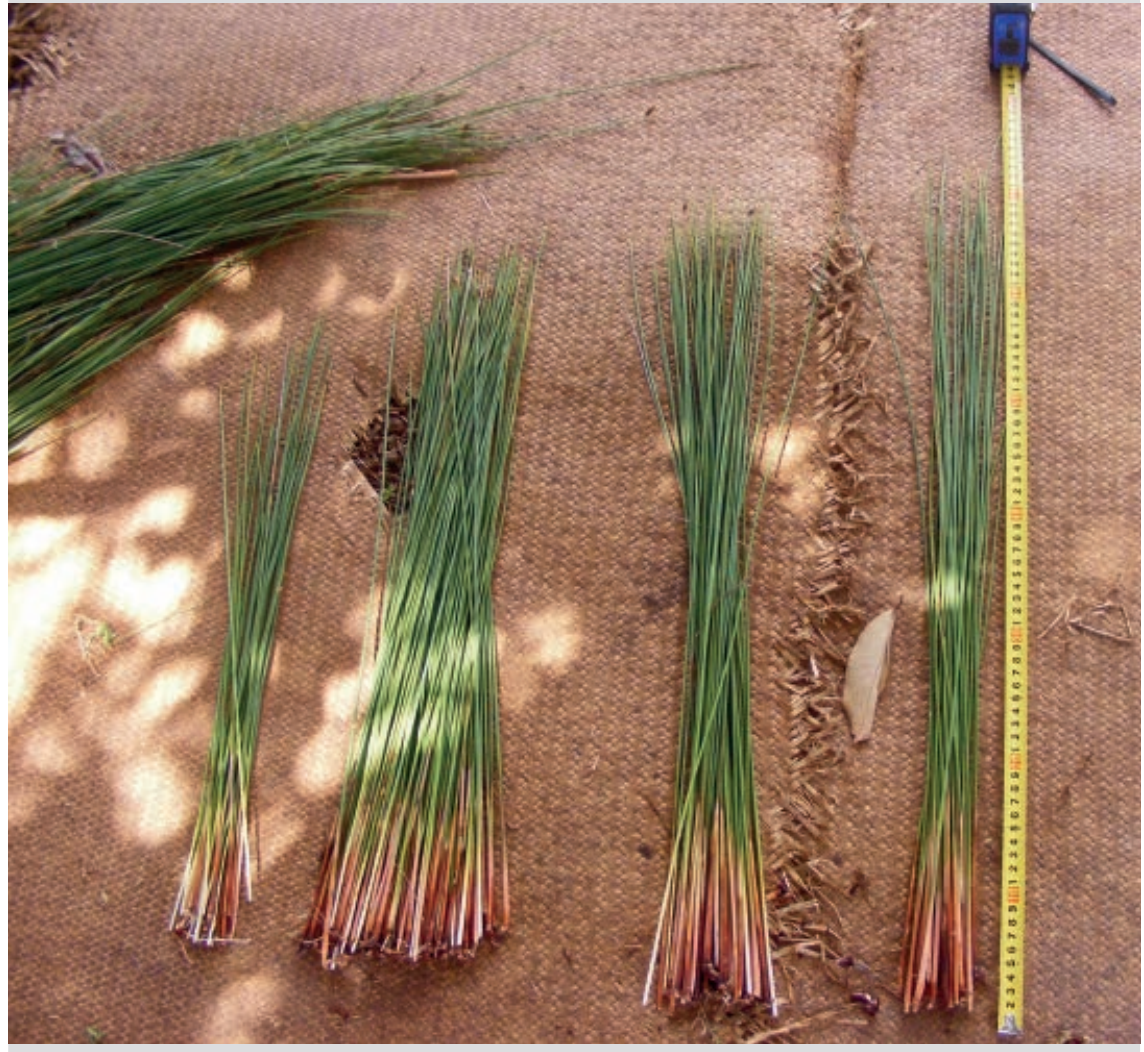

Photo 4.

Répartition des tiges prélevées dans les marais, par classes de hauteur.

Photo S. Guillaud.

\section{Dégradation du mahampy}

Pour estimer l'état de la dégradation de la ressource, les traces de prélèvements et de feux ainsi que la présence de zébus, Bos indicus, et de chemins de collecte ont été répertoriées à l'échelle du marais.

\section{Étude socio-économique de la vannerie}

Les enquêtes ont été menées auprès des ménages ruraux vivant en périphérie ou au sein même de la Réserve spéciale de Manombo et de la forêt d'Agnalazaha. La filière vannerie a été étudiée dans les marchés ruraux de Manombo et Mahabo, situés à proximité des deux espaces naturels et au sein des marchés urbains de Farafangana et de Vangaindrano (figure 1).

Pour obtenir des informations sur les aspects socio-économiques et culturels de la vannerie dans la région, trois groupes cibles ont été préidentifiés : les commerçantes de vannerie, les vannières et les chefs de ménage.

Toutes les enquêtes, de type anonyme, ont été réalisées selon la méthode du questionnaire ouvert, qui consiste à proposer une liste précise de questions fermées ou ouvertes auxquelles l'enquêté peut répondre aussi longtemps qu'il le désire.

\section{Productivité}

La productivité de chaque peuplement de L. mucronata a été estimée par la densité, la hauteur et le diamètre moyen des tiges vivantes (stades de développement 1 et 2) au sein des UE. Toutes les tiges vivantes de mahampy ont été extraites de la surface du quadrat. Ces tiges ont été réparties par classe de hauteur, à l'aide d'un mètre ruban (photo 4). La séparation des tiges par catégorie de hauteur s'est opérée de façon à obtenir des intervalles de classes de $10 \mathrm{~cm}$. Le diamètre a été mesuré avec un pied à coulisse sur $10 \%$ de l'effectif des tiges de chaque classe de hauteur.

Ces données ont permis par la suite d'envisager la disponibilité de cette ressource face aux prélèvements des vannières, dont les critères de sélection des tiges se basent sur leur grandeur et leur grosseur.

Un test d'égalité des moyennes pondéré à deux critères de classification et à modèle hiérarchisé mixte a été établi afin de pouvoir comparer les diamètres et les hauteurs moyens des tiges des trois marais. Une analyse de la variance à un critère de classification a été utilisée pour comparer la densité en tiges des marais. Par ailleurs, cette estimation a été complétée en utilisant les données de l'étude menée par IKUSIMA (1978), qui propose différents moyens de mesurer la productivité d'un peuplement de Lepironia mucronata.
Les données récoltées ont été de deux types. Les premières, cognitives, ont permis d'obtenir des informations d'ordre quantitatif. Le constat s'est fait sur la base d'une identification chiffrée, utilisant des mots interrogatifs tels que : où, combien, quand. Le second type de données récoltées était de type affectif, mettant en évidence les motivations et les stratégies appliquées par les acteurs et permettant de comprendre certains phénomènes.

Afin de mener des enquêtes auprès des vannières et des chefs de ménage, la liste des villages a été établie pour chaque zone avec l'aide du mpanzaka (roi local) et du cheffokontany (autorité administrative d'un groupe de villages). Un seul ménage, choisi au hasard, fut interrogé par village. Au total, onze femmes et leurs époux ont été enquêtés dans chaque zone, représentant au total 66 personnes. À travers les enquêtes auprès des vannières (annexe 1 ), l'organisation pratique de la collecte et de la transformation des tiges ainsi que la gestion de cette ressource ont été étudiées. Les dimensions sociale et économique de la vannerie (prix de revient, bénéfices...) ont également été analysées lors de ces enquêtes. Les enquêtes auprès des chefs de ménage (annexe 2) ont permis de connaître l'organisation productive et la situation socio-économiques des familles. Quant aux commercantes, treize ont été choisies au hasard et interrogées dans les deux marchés urbains de la région (annexe 3 ). 


\section{Résultats}

\section{Étude de la végétation}

Stade de développement des tiges de Lepironia mucronata

Le tableau I présente pour chacun des marais le pourcentage de tiges dans chaque stade de développement.

\section{Mesures de productivité}

Les mesures de la productivité des marais, exprimées par la hauteur, le diamètre et la densité des tiges vivantes, se trouvent dans le tableau II et sont présentées respectivement dans les figures 2, 3 et 4 .

Selon le test d'égalité des moyennes utilisé, des différences hautement significatives entre les trois marais sont détectées pour la hauteur moyenne $(F=122,7$; $d l=2$; $P$ < $0,001)$. Cependant, la hauteur moyenne des tiges entre les quadrats de chaque marais ne diffère pas de manière significative $(F=1,1 ; d l=60 ; P=0,260)$.

Les mêmes conclusions peuvent être tirées pour les diamètres moyens des tiges entre marais $(F=96,06 ; \mathrm{dl}=2$; $P<0,001)$ et au sein de chaque marais $(F=1,08 ; d l=60$; $P=0,347$ ), les diamètres moyens des tiges entre quadrats de chaque marais ne différant pas de manière significative. Une transformation de type logarithmique a été appliquée à la variable "densité de tiges » afin de répondre aux conditions d'application. En conséquence, il est constatable que des différences hautement significatives entre les trois marais sont observées au niveau du logarithme de la densité moyenne de tiges $(F=17,8 ; d l=2 ; P<0,001)$.
La comparaison des moyennes permet de constater que les densités moyennes de tiges d'Agnanato et d'Apilatenitry ne diffèrent pas entre elles. La densité moyenne de tiges du marais d'Amparahibe diffère par contre de manière significative des deux autres.

Il est possible de conclure que le marais d'Apilatenitry possède des tiges de mahampy significativement plus hautes tandis qu'Amparahibe présente les tiges les plus grosses et les plus denses. Quant aux tiges d'Agnanato, elles sont les plus petites et les plus fines des trois marais. Par ailleurs, les tiges de mahampy sont toutes homogènes en termes de hauteur, diamètre et densité au sein de chaque peuplement.

\section{Dégradation des peuplements de Lepironia mucronata}

Les pressions exercées au sein des trois marais sont nombreuses. À Agnanato, la collecte massive des tiges pour la vannerie et le réseau de sentiers occasionné, le passage de zébus (traces de passage et déjections) et les feux accidentels en saison sèche sont les principales pressions anthropiques relevées. Sur toute la surface du marais, des tiges arrachées avec leur rhizome et abandonnées sur place ont été observées. Les pressions anthropiques sur Amparahibe concernent surtout la collecte massive des tiges (réseau de sentiers et tiges arrachées et abandonnées) même si quelques traces de passage d'animaux et des zones brûlées y ont également été constatées. À Apilatenitry, les seules pressions anthropiques observées sur le marais sont les traces de prélèvements et le réseau de sentiers résultants.

Tableau I.

Stades de développement des tiges de Lepironia mucronata.

\begin{tabular}{l|c|c|c|c|c|} 
& $\begin{array}{c}\text { Tiges en } \\
\text { développement (\%) }\end{array}$ & Écart-type & $\begin{array}{c}\text { Tiges en } \\
\text { floraison (\%) }\end{array}$ & Écart-type & $\begin{array}{c}\text { Tiges mortes ou } \\
\text { en sénescence (\%) }\end{array}$ \\
\hline Agnanato & 38,26 & 18,07 & 8,56 & 9,94 & 53,17 \\
\hline Amparahibe & 39,61 & 13,03 & 20,2 & 17,46 & 40,19 \\
\hline Apilatenitry & 53,84 & 16,09 & 4,61 & 3,79 & 41,53 \\
\hline
\end{tabular}

Tableau II.

Mesures de hauteur, de diamètre et de densité des tiges vivantes dans les trois marais.

\begin{tabular}{|c|c|c|c|c|c|c|c|c|c|}
\hline & & Hauteur (cm) & & & Diamètre $(\mathrm{cm})$ & & Dens & ité (nombre ti & $\left.\operatorname{ges} / \mathrm{m}^{2}\right)$ \\
\hline & Agnanato & Amparahibe & Apilatenitry & Agnanato & Amparahibe & Apilatenitry & Agnanato & Amparahibe & Apilatenitry \\
\hline Moyenne estimée & 59,7 & 81,8 & 86,6 & 0,36 & 0,476 & 0,45 & 213 & 421 & 140 \\
\hline Écart-type estimé & 5,74 & 5,92 & 4,97 & 0,0299 & 0,02408 & 0,0209 & 81,6 & 271,5 & 54,4 \\
\hline $\begin{array}{l}\text { Erreur } \\
\text { d'échantillonnage }\end{array}$ & 8,38 & 11,98 & 20,98 & 0,0508 & 0,0698 & 0,108 & 35,3 & 109,68 & 31,42 \\
\hline $\begin{array}{l}\text { Intervalle de confiance } \\
\text { de la moyenne }\end{array}$ & {$[51,3-68,1]$} & {$[69,9-93,8]$} & {$[65,7-107,6]$} & {$[0,309-0,41]$} & {$[0,406-0,545]$} & {$[108,6-171,4]$} & {$[177,7-248,3]$} & [311,3-530,6] & {$[108,6-171,4]$} \\
\hline
\end{tabular}




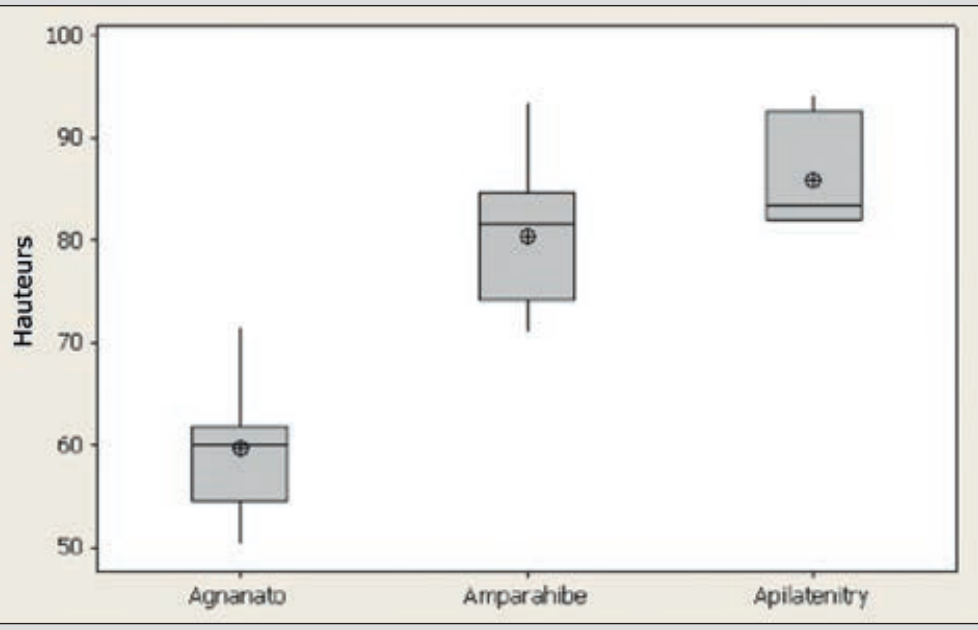

Figure 2 :

Répartition des hauteurs de tiges, dans les trois marais.

Source : S. Guillaud.

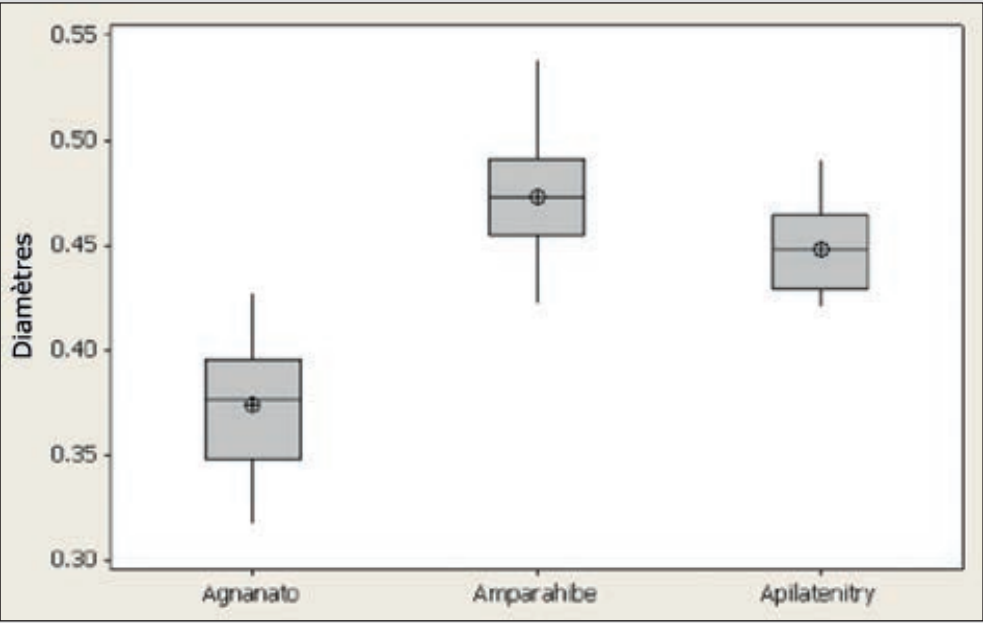

Figure 3 :

Répartition des diamètres de tiges, dans les trois marais.

Source : S. Guillaud.

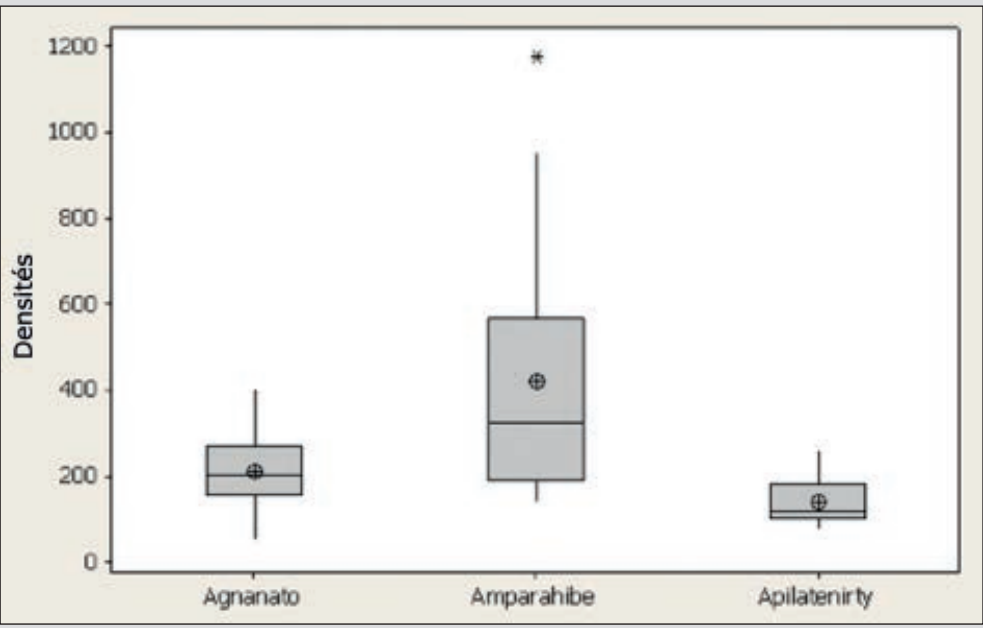

Figure 4 :

Répartition des densités de tiges, dans les trois marais.

Source : S. Guillaud.

\section{Analyse de la filière vannerie traditionnelle et des retombées économiques dans la région}

Exploitation et gestion traditionnelle du mahampy

Pour rappel, les deux premiers marais étudiés, Agnanato et Amparahibe, sont situés en pays Antesaka tandis que le troisième, Apilatenitry, se trouve en pays Antevato.

Chez les Antevato, un dina (règle villageoise) important régit la collecte du mahampy en interdisant à quiconque de vendre des tiges brutes (rambo). En outre, le droit d'accès aux marais de cette zone est de type ethnique, et il est interdit à toute personne non Antevato de pénétrer dans ces marais pour prélever des tiges, sous peine de devoir payer une amende, prélevée par le chef local, appelé Mpanzaka. Il s'agit donc, d'après la théorie des mâ̂trises foncières de LE ROY et al. (1996), adaptée par VERMEULEN et CARRIÈRE (2001), d'une maîtrise exclusive de la ressource, incluant le droit d'accès, d'extraction, de gestion et d'exclusion selon des modalités de cogestion de type externe, car elles concernent l'ethnie Antevato, tous clans confondus.

Dans le cas des marais d'Agnanato et d'Amparahibe, en pays Antesaka, l'accès à la ressource est interdit pour les personnes extérieures à la commune de Mahabo. Les modalités de cogestion sont de type public (dans les limites de la commune) et la maîtrise de la ressource est prioritaire, concernant le droit d'accès et d'extraction.

\section{La vannerie et sa filière économique}

La vannerie est une activité principalement féminine dont le savoir-faire se transmet de génération en génération. Les femmes se chargent de la cueillette des tiges (photo 5), de leur tressage (photo 6) et de la vente des produits vannés (photo 7).

Les principales étapes de la transformation des tiges sont le séchage, le découpage en lanières, la coloration et enfin le tressage.

Quant aux principaux objets vannés, ils se déclinent en nattes, paniers et haro (simple panier sans anses permettant de contenir et de transporter les denrées alimentaires). Les femmes de certains fokontany se sont parfois spécialisées dans la confection d'un type d'objet. Ainsi, les femmes du fokontany de Mahabo et de Vohimasy font essentiellement des paniers, alors que celles du fokontany de Karimbelo fabriquent surtout des nattes ; mais dans les deux cas, ces femmes confectionnent aussi des haro.

Lors des enquêtes, la quantité moyenne de tiges nécessaires à la confection des objets a été calculée. Elle est de 260 tiges (écart-type de 41) pour un panier, 370 pour un grand haro (écart-type de 70) et 1760 pour une natte constituée de trois parties (écart-type de 95). La confection de nattes ou de paniers requiert des tiges d'une hauteur minimale de 90 à $100 \mathrm{~cm}$. 


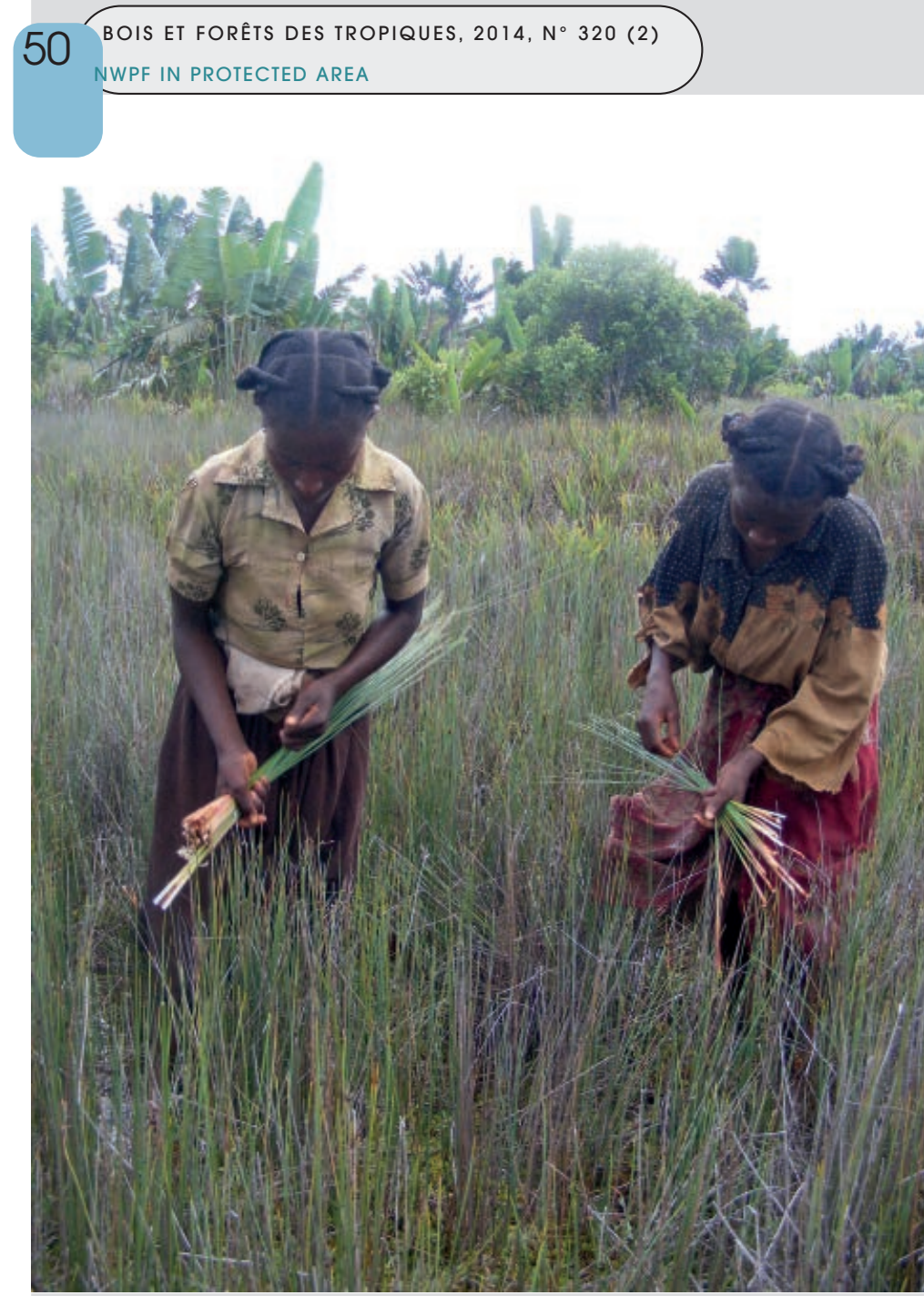

Photo 5.

Cueillette des tiges de mahampy dans le marais d'Amparahibe. Photo S. Guillaud.

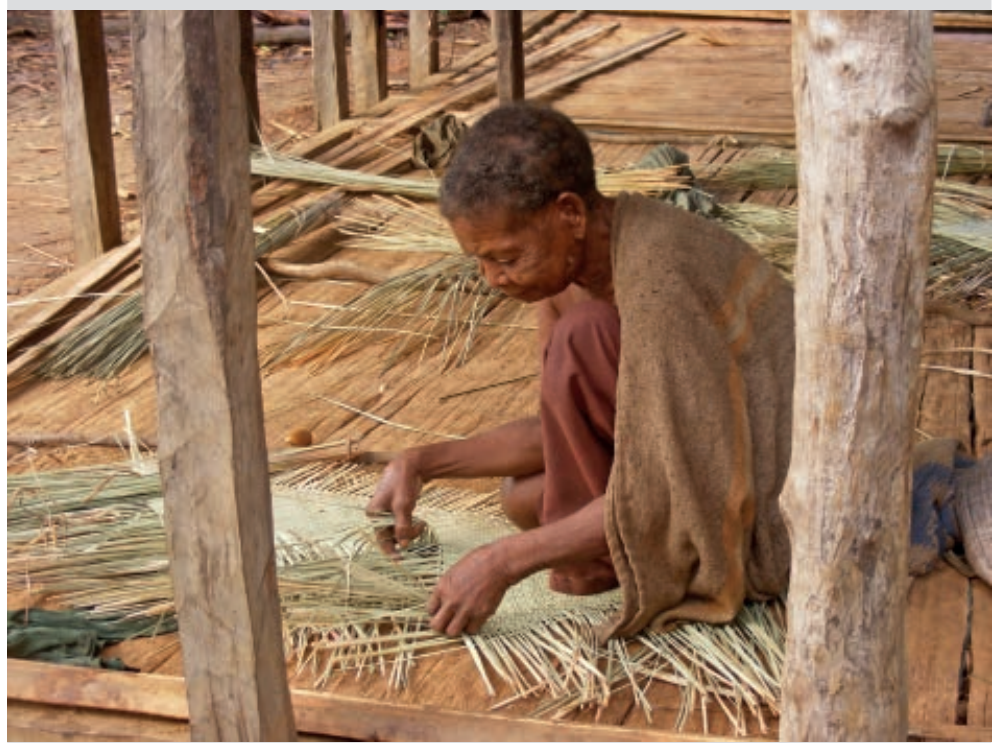

Photo 6.

Tressage des tiges pour la fabrication d'une natte. Photo S. Guillaud.

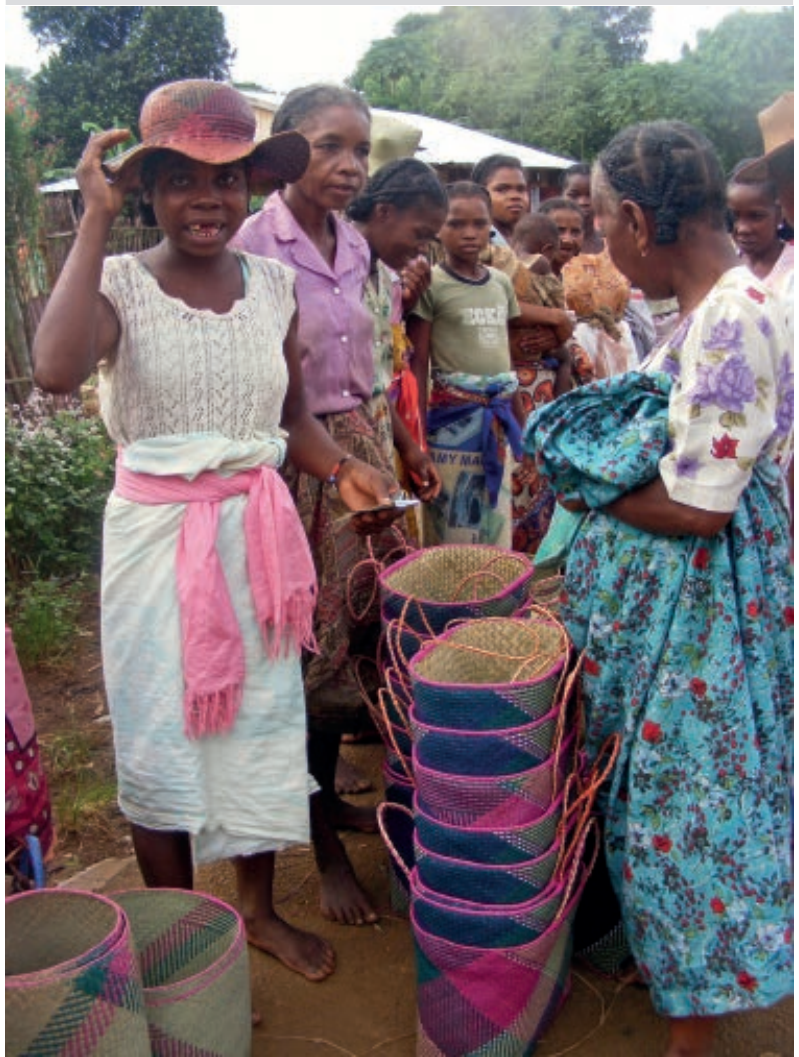

Photo 7.

Vente de la vannerie dans le marché de brousse de Mahabo. Photo S. Guillaud.

L'organisation de la filière vannerie dans la région d'Atsimo-Atsinanana est relativement simple (figure 5). Deux marchés urbains (amprositry) existent : le marché de Farafangana et le marché de Vangaindrano. Distantes de $75 \mathrm{~km}$, ces deux villes sont connectées par la Nationale 12, le long de laquelle se trouvent quelques marchés de brousse (tsena) où a lieu le commerce de vannerie. Les fruits, les légumes et les poissons de rivière sont les autres principaux produits vendus dans ces marchés.

L'accès à la ressource détermine deux types distincts de vannières : celles pouvant accéder aux marais et transformer les tiges qu'elles ont collectées elles-mêmes, et celles devant les acheter car n'ayant pas accès à cette matière première. Dans ce dernier cas, les vannières se les procurent auprès de femmes d'autres fokontany, en les achetant sous forme brute (rambo) dans les marchés de brousse (photo 8).

Trois acteurs principaux entrent dans la filière vannerie : les vannières (mpandrary), les commerçantes (client lamaky) et les consommateurs. Comme ces derniers ne se rendent jamais en brousse pour y acheter de la vannerie, les commerçantes sont donc des intermédiaires permanentes. Elles se rendent régulièrement aux marchés de brousse, y achètent divers articles aux vannières et les revendent ellesmêmes dans les marchés urbains (photo 9). En outre, il existe quelques rares commerçantes venant d'autres régions qui achètent la vannerie sur les marchés locaux et la revendent dans d'autres marchés du pays. D'autres acteurs indirects entrent également dans la filière vannerie. II s'agit des 
Vannières ayant accès aux marais :

elles tressent et peuvent, en plus de leur propre vannerie, vendre des tiges brutes au marché local.

2

Vannières n'ayant pas accès aux marais :

elles doivent ainsi acheter des tiges brutes pour leur vannerie qu'elles vendront ensuite au marché local.

\section{3}

Commerçantes de vannerie régionales :

elles achètent la vannerie sur les marchés locaux et la revendent dans les marchés urbains de la région.

\section{4}

Commerçantes de vannerie nationales :

elles achètent la vannerie sur les marchés locaux et la revendent dans d'autres marchés en dehors de la région.

\section{5}

Détaillants des consommations intermédiaires : raphia et colorants.

\section{6}

Transporteurs (taxi-brousse), propriétaires des pièces de stockage et porteurs des marchandises : des commerçantes, du lieu de stockage au marché.

Figure 5 :

Organisation de la filière vannerie locale et régionale.

détaillants de raphia et de colorants nécessaires à la confection de certains objets vannés, des porteurs aidant les commerçantes à transporter leur vannerie de leur emplacement de vente jusqu'à leur lieu de stockage, des propriétaires de ces pièces de stockage et des transporteurs routiers (coopératives de taxi-brousse) qui permettent aux commerçantes d'effectuer les trajets de la ville jusqu'en brousse.

\section{Prix de revient et bénéfices engendrés par les vannières}

La confection de vannerie et la vente des produits finis sont tributaires du calendrier rizicole qui diffère entre les ethnies. Chez les Antevato, peuple de pêcheurs, on distingue deux périodes principales : les mois de " soudure" de juillet à mars, où les travaux agricoles demandent peu de main-d'œuvre (entretien des rizières et culture d'autres denrées alimentaires comme le manioc) et les mois de récolte du riz, d'avril à juin. Le calendrier cultural des Antesaka comprend quant à lui une seconde période de récolte du riz, de novembre à décembre.

Pendant la période de soudure, toutes les femmes pratiquent la vannerie de façon intensive. Celle-ci inonde ainsi les marchés de la région et les prix offerts par les commerçantes baissent systématiquement. Au contraire, en période de récolte du riz, les quantités d'objets offertes sur les marchés diminuent drastiquement et leurs prix de vente augmentent en conséquence.

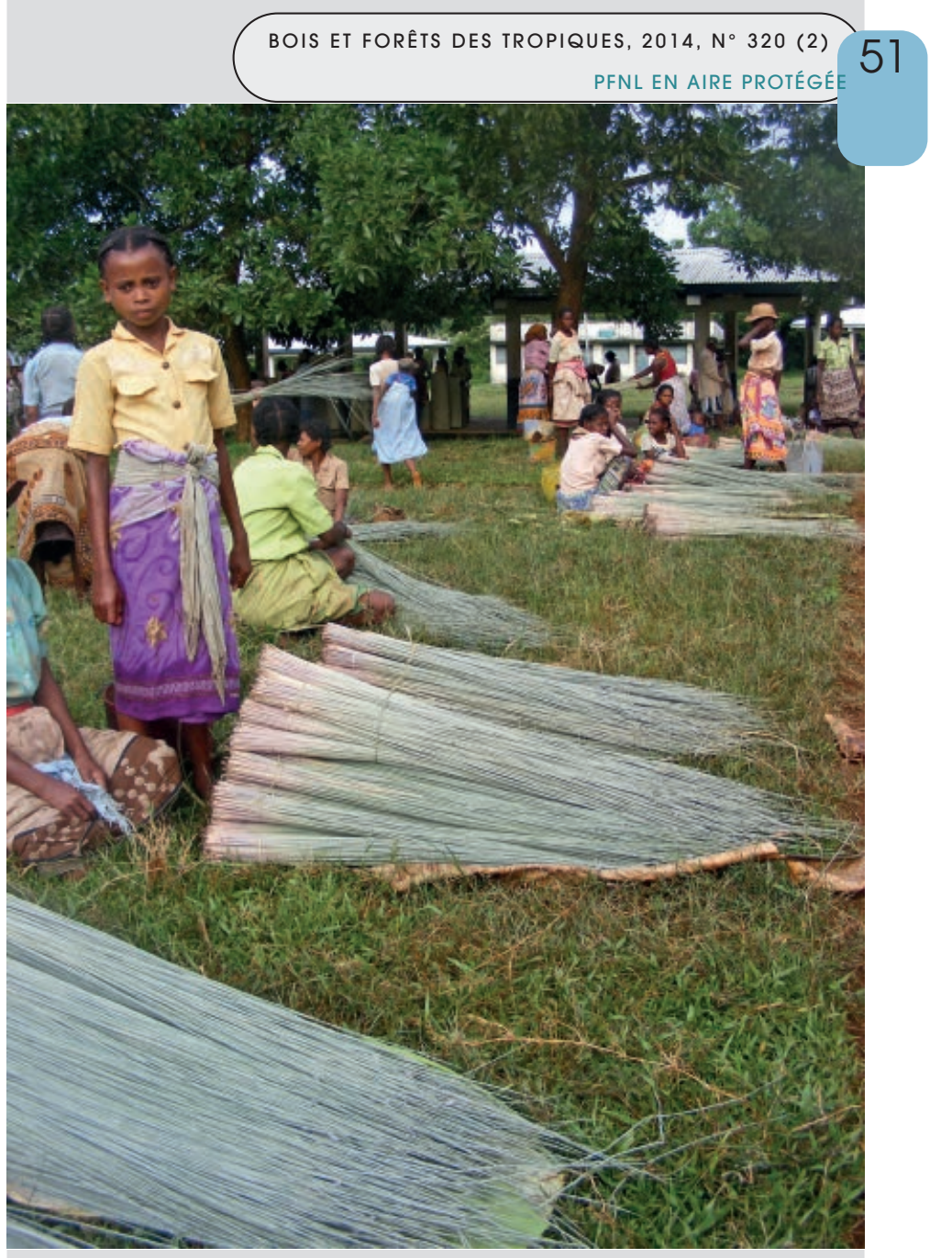

Photo 8.

Vente de tiges de mahampy brutes dans le marché de brousse de Mahabo à destination des vannières n'ayant pas accès aux marais.

Photo S. Guillaud.

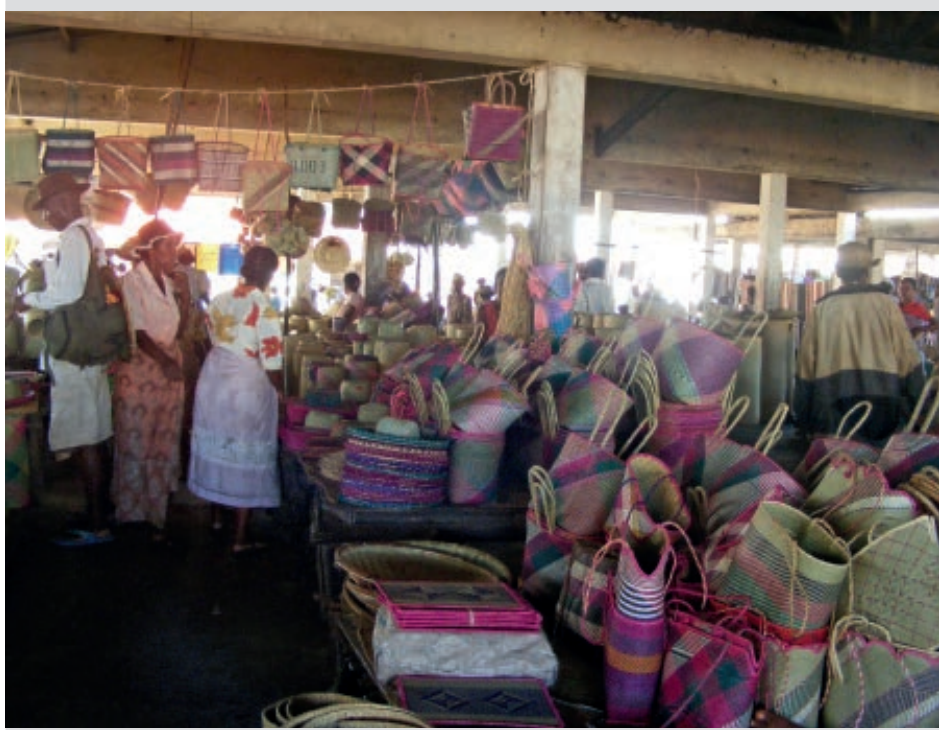

Photo 9.

Partie du marché de Farafangana où a lieu la vente de la vannerie, à destination des ménages urbains. Photo S. Guillaud. 
Tableau III.

Coût de revient moyen, prix de vente moyen et bénéfices moyens engendrés par la vente d’objets et par période.

\begin{tabular}{l|c|c|c|c|c|} 
& $\begin{array}{c}\text { Coût de revient } \\
\text { moyen }\end{array}$ & $\begin{array}{c}\text { Prix de vente } \\
\text { moyen : période } \\
\text { de récolte du riz }\end{array}$ & $\begin{array}{c}\text { Prix de vente } \\
\text { moyen : période } \\
\text { de soudure }\end{array}$ & $\begin{array}{c}\text { Bénéfice moyen : } \\
\text { période de récolte } \\
\text { du riz }\end{array}$ & $\begin{array}{c}\text { Bénéfice moyen : } \\
\text { période } \\
\text { de soudure }\end{array}$ \\
\hline Panier & $900 \operatorname{Ar}(0,36 €)$ & $2500 \operatorname{Ar}(1 €)$ & $2000 \operatorname{Ar}(0,85 €)$ & $1600 \operatorname{Ar}(0,65 €)$ & $1100 \operatorname{Ar}(0,50 €)$ \\
\hline Haro & 0 & $800 \operatorname{Ar}(0,32 €)$ & $500 \operatorname{Ar}(0,20 €)$ & $800 \operatorname{Ar}(0,32 €)$ & $500 \operatorname{Ar}(0,20 €)$ \\
\hline Grande natte & 0 & $7500 \operatorname{Ar}(3 €)$ & $5600 \operatorname{Ar}(2,25 €)$ & $7500 \operatorname{Ar}(3 €)$ & $5600 \operatorname{Ar}(2,25 €)$ \\
\hline Petite natte & 0 & $4200 \operatorname{Ar}(1,70 €)$ & $3000 \operatorname{Ar}(1,20 €)$ & $4200 \operatorname{Ar}(1,70 €)$ & $3000 \operatorname{Ar}(1,20 €)$ \\
\hline
\end{tabular}

Les différents coûts de revient et les bénéfices, comme l'indique le tableau III, dépendent également du prix d'achat des produits intermédiaires (colorants et raphia).

Dans le cas des paniers, le coût de fabrication dépend d'abord de l'origine des tiges, c'est-à-dire si elles ont été collectées par la vannière elle-même ou si celle-ci a dû les acheter. Cependant, la plupart des paniers sont confectionnés dans la commune de Mahabo, qui ne comprend plus aucun marais, avec des tiges de mahampy suffisamment longues. Ces femmes dépendent donc de tiges provenant de marais plus éloignés et revendues dans les marchés de brousse. Un paquet de tiges brutes de mahampy (rambo) permettant la confection d'un panier coûte $300 \operatorname{Ar}(0,12 €)$. En outre, le coût de fabrication d'un panier dépend de la quantité de colorant et de raphia qu'il nécessite. Un paquet de colorant en poudre coûte $100 \operatorname{Ar}(0,04 €)$ et il en faut généralement trois pour colorer complètement un panier. La quantité de raphia nécessaire à un panier coûte environ $300 \mathrm{Ar}$. La confection des haro nécessite peu ou pas de colorant et très peu de raphia. Les tiges utilisées dans les haro sont habituellement collectées par les vannières ellesmêmes et n'interviennent donc pas dans le coût de revient. Celui-ci est donc souvent nul. De même, les nattes ne nécessitent ni colorant, ni raphia et les tiges sont toujours collectées par les vannières.

Généralement, une vannière peut confectionner en une semaine soit une natte, soit cinq paniers, soit sept à dix haro. Si le coût du travail est internalisé, en se basant sur le salaire horaire minimum pour les travailleurs non agricoles de $396 \operatorname{Ar}(0,16 €)$ (URFER, 2009), toutes les vannières vendent à perte. En effet, en estimant qu'une journée de travail équivaut à 8 heures et une semaine de travail à 6 jours, le coût du travail peut être évalué à $7,08 €$ pour la confection d'une grande natte, à $1,41 €$ pour un panier et à $0,88 €$ pour un haro. Comme en témoigne le tableau III, ces coûts sont largement supérieurs au prix de vente des produits vannés.

Avec l'argent issu de la vente de la vannerie, ces femmes rachètent immédiatement le matériel nécessaire à la confection des articles suivants (tiges, raphia, colorants) et des biens de consommation courante.

\section{Coûts et revenus des commerçantes de vannerie}

Les commerçantes prennent une marge commerciale constante de 100 à $200 \operatorname{Ar}(0,04$ à $0,08 €)$ sur les haro, de 500 à $1000 \operatorname{Ar}(0,20$ à $0,40 €)$ sur les nattes, et de 300 à $700 \operatorname{Ar}(0,12$ à $0,28 €)$ sur les paniers. Ces marges leur permettent de faire face à leurs frais de transport, à la taxe journalière et la redevance communale pour leurs emplacements au marché, à la location d'un lieu de stockage nocturne pour leurs marchandises et au paiement des porteurs, chargés d'amener cette marchandise du marché au lieu de stockage (tableau IV).

Ainsi, un panier vendu $3000 \mathrm{Ar}$ en ville présente une valeur ajoutée moyenne répartie entre la vannière (45\%), les détaillants de produits intermédiaires (30\%) et les commerçantes (bénéfice et frais répartis sur les $25 \%$ restant).

Tableau IV.

Frais supportés par les commerçantes de vannerie dans les deux marchés régionaux.

\begin{tabular}{|l|r|r|}
\hline Frais & \multicolumn{1}{c}{$\begin{array}{c}\text { Commerçantes } \\
\text { de Farafangana }\end{array}$} & $\begin{array}{c}\text { Commerçantes } \\
\text { de Vangaindrano }\end{array}$ \\
\hline Trajet aller-retour marché Mahabo & $6000 \operatorname{Ar}(2,4 €)$ & $4000 \operatorname{Ar}(1,6 €)$ \\
\hline Trajet aller-retour marché Manombo & $4000 \operatorname{Ar}$ & $6000 \operatorname{Ar}(2,4 €)$ \\
\hline Taxe journalière & $100 \operatorname{Ar}(0,04 €)$ & $200 \operatorname{Ar}(0,08 €)$ \\
\hline Taxe annuelle & $20000-40000 \operatorname{Ar}(8-16 €)$ & $2000 \operatorname{Ar}(0,80 €)$ \\
\hline Location mensuelle salle de stockage & $0-3000 \operatorname{Ar}(0-1,2 €)$ & $0-3000 \operatorname{Ar}(0-1,2 €)$ \\
\hline Porteur journalier (aller-retour) & $800 \operatorname{Ar}(0,32 €)$ & $800 \operatorname{Ar}(0,32 €)$ \\
\hline
\end{tabular}




\section{Une activité économique d'importance}

Les activités économiques des Antesaka, peuple d'agriculteurs, se limitent le plus souvent à de l'aquiculture d'autosubsistance. Très peu parviennent à dégager des surplus agricoles qu'ils peuvent vendre et ces revenus sont faibles et irréguliers. Quant aux Antevato, peuple de pêcheurs, certains ménages bénéficient de quelques revenus engendrés par la pêche, mais l'autosuffisance en riz y est encore plus faible que chez les Antevato. Dans les deux cas, la vannerie consitue la source principale de revenus monétaires des ménages enquêtés. Les apports financiers fixes et réguliers qu'elle fournit permettent aux femmes d'acheter les produits de consommation de base comme le sel, l'huile, le savon, le sucre, ou encore le pétrole pour s'éclairer. Vingtsix ménages sur les 33 enquêtés considèrent la vannerie comme leur unique source de revenus, leur permettant ainsi de faire face aux frais de scolarité des enfants et à leurs besoins courants. De ce fait, la vannerie est généralement pratiquée tout au long de l'année. Parmi les 33 vannières enquêtées, huit femmes cessaient complètement la vannerie, seize diminuaient légèrement la quantité d'objets fabriqués tandis que neuf continuaient à produire autant d'objets qu'en période de soudure.

La manière dont la vannerie diminue pendant la période de récolte renseigne la richesse du ménage en surfaces agricoles. Si les quantités d'objets tressés diminuent faiblement, cela signifie que le ménage possède peu de rizières et a besoin de peu de main-d'œuvre pour faire face aux travaux agricoles. En plus, ce type de ménage doit maintenir ses rentrées monétaires afin de subvenir, en plus de ses besoins courants, à ses besoins alimentaires.

\section{Fréquence de la collecte des tiges}

La fréquence avec laquelle les femmes partent récolter le mahampy dépend de leurs besoins personnels en tiges et donc du moment de l'année.

Les besoins en tiges dépendent donc du nombre et du type d'objets confectionnés, variables selon chaque vannière et selon les années (légère variation de la durée des périodes de soudure ou de récolte). Le tableau $\mathrm{V}$ reprend quelques scénarios possibles dans le marais d'Amparahibe, issus des observations de terrain.

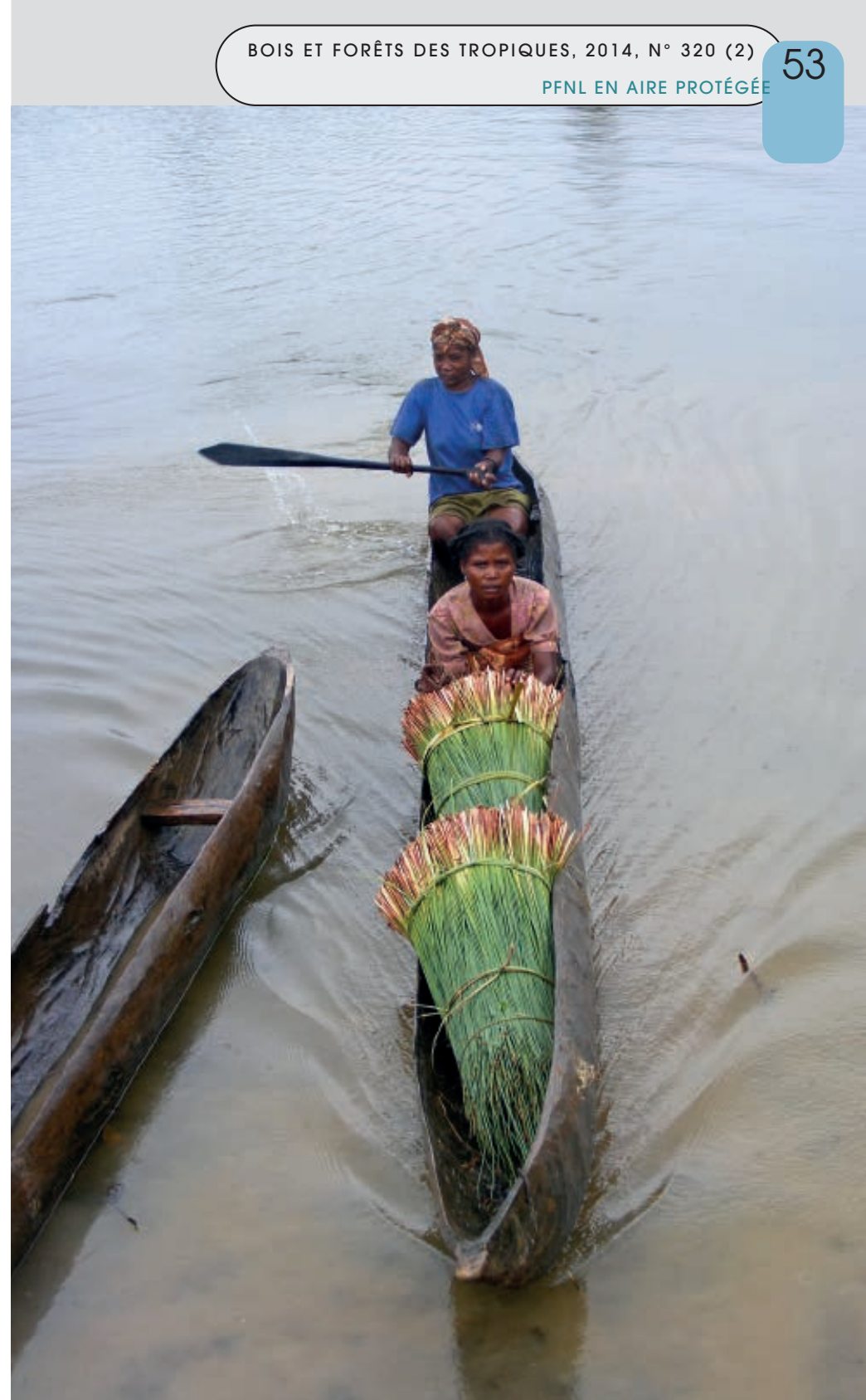

Photo 10.

Transport des tiges.

Photo S. Guillaud.

Tableau V.

Scénarios de confection de vannerie, de besoin en tiges et de surface correspondante.

Scénario

$$
\begin{aligned}
& \text { Périodes de soudure } \\
& \text { (durée : } 8 \text { mois) }
\end{aligned}
$$
Quantité mensuelle Nombre total de de produits vannés tiges nécessaire

\author{
Périodes de récolte \\ (durée : 4 mois) \\ Quantité mensuelle Nombre total de \\ de produits vannés tiges nécessaire
}

Bilan annuel

Besoin annuel en tiges
Surface nécessaire

\begin{tabular}{|c|c|c|c|c|c|c|}
\hline Vannière 1 & 4 nattes & 56320 & 0 (arrêt de la vannerie) & 0 & 56320 & 133,8 \\
\hline Vannière 2 & 36 haro & 106560 & 4 haro & 5920 & 112480 & 267,2 \\
\hline Vannière 3 & 4 nattes & 56320 & 3 haro & 4440 & 60760 & 144,3 \\
\hline
\end{tabular}
à Amparahibe $\left(\mathrm{m}^{2}\right)$ 
Les femmes prélèvent des tiges quasiment toute l'année dans le marais d'Apilatenitry alors que, dans le marais d'Amparahibe et d'Agnanato, la plupart d'entre elles diminuent la fréquence des prélèvements en période de récolte du riz.

Généralement, une femme collecte la quantité maximale qu'elle est capable de transporter (photo 10) et retournera en collecter dès que sa réserve de tiges sera épuisée.

\section{L'état de la ressource à dire d'acteurs}

Le marais d'Agnanato a été fortement exploité par le passé par les femmes des fokontany de Mahabo et de Vohimasy et ne présente désormais que des tiges de longueur et de diamètre restreints. D'après les femmes interrogées, la dégradation progressive du mahampy s'explique par les incendies et par la pression croissante de la collecte. Désormais, la faible hauteur des tiges d'Agnanato (hauteur moyenne de 59,7 cm) limite fortement leur usage et seuls des haro peuvent être conçus.

Autour du marais d'Amparahibe, dans le fokontany de Karimbelo, toutes les femmes interrogées affirment que les tiges de mahampy de ce marais diminuent quantitativement et qualitativement au cours du temps. Elles mentionnent également la surexploitation des tiges par un nombre croissant de vannières.

Autour du marais d'Apilatenitry, les femmes exploitant le marais d'Apilatenitry délivrent le même constat sur l'état du mahampy que les femmes du fokontany de Karimbelo et mentionnent les mêmes facteurs responsables de sa dégradation.

Par ailleurs, la majorité des femmes mentionnent le fait que la période de repos des marais créée par la période de récolte du riz, d'avril à juin, permet aux tiges de pousser. Cette courte " jachère involontaire » des marais a lieu juste après la saison des pluies, facteur complémentaire à la reprise de la croissance des tiges.

\section{Discussion et conclusion}

Les différences entre les peuplements de Lepironia mucronata des trois marais étudiés sont attribuables d'une part à des facteurs environnementaux (effet site), comme le type et la qualité du sol, le niveau d'inondation moyen annuel, ou encore le $\mathrm{pH}$, et d'autre part à des facteurs anthropiques. Les facteurs environnementaux n'ayant pas pu être mesurés dans cette étude, seuls les facteurs anthropiques peuvent être interprétés. Cependant, d'après les dires de toutes les personnes enquêtées, le marais d'Agnanato présentait dans le passé une ressource en tiges proche du marais d'Amparahibe. Le meilleur état général du mahampy au sein des marais d'Apilatenitry et d'Amparahibe s'explique donc en partie par leur situation dans la Réserve spéciale de Manombo. Ils y jouissent en effet d'une certaine protection face aux pressions anthropiques. Les règlements contraignants appliqués tant bien que mal dans la zone ont probablement contribué à contenir l'expansion des cultures sur brûlis, contrairement au cas du marais d'Agnanato. La gestion traditionnelle qui régule l'exploitation d'Apilatenitry participe également à préserver la ressource.
Grâce aux résultats issus des enquêtes et à notre estimation de la productivité des marais (exprimée par la densité, le diamètre et la hauteur des tiges), il est possible de proposer une première approche de la quantité totale de tiges prélevées annuellement par femme et de la surface de marais répondant à ces besoins. De cette façon, il est possible de tenir un discours sur les possibilités d'exploitation des marais.

À titre d'exemple, une femme Antesaka qui confectionne cinq paniers par semaine en pleine période de soudure (durée moyenne de quatre mois) et deux paniers par semaine en pleine période de récolte du riz (durée moyenne de deux mois) nécessitera respectivement 20800 et 4160 tiges pour chacune des périodes en question. Cela représente environ 50000 tiges annuelles, lorsque l'année est caractérisée par une double période de récolte du riz. Dans le cas du marais d'Amparahibe (densité moyenne de 421 tiges vivantes par mètre carré), cela correspond à une surface de 118,7 m².

Ce type d'estimation, certes grossière, permet par exemple, lors de la création d'une plantation de mahampy ou d'une réorganisation de l'accès à la ressource, de connaître la surface minimale nécessaire à chaque vannière pour répondre à ses besoins en tiges.

Une autre approche consiste à confronter la capacité de renouvellement des tiges d'un marais à la pression exercée sur ce dernier afin d'infirmer ou confirmer la surexploitation de la ressource par la vannerie.

Pour cela, il est nécessaire d'estimer d'abord le taux de renouvellement naturel des tiges de $L$. mucronata grâce à des facteurs tels que la croissance, le taux d'émergence de nouvelles tiges et la répartition globale des classes de hauteur au sein du marais. En se basant sur l'estimation d'un nombre moyen de cinq tiges vivantes par rhizome (IKUSIMA, 1978), le marais d'Amparahibe produit donc environ 84 rhizomes $/ \mathrm{m}^{2}$. En partant de ces dernières estimations et $\mathrm{du}$ taux d'émergence de nouvelles tiges par rhizome (une nouvelle tige toutes les huit semaines) (IKUSIMA, 1978), Amparahibe produirait 504 nouvelles tiges $/ \mathrm{m}^{2} / \mathrm{an}$.

En multipliant ce nombre par la surface de ce marais (34 $000 \mathrm{~m}^{2}$ ), et en considérant qu'il en est entièrement couvert, il est possible d'y estimer la quantité totale de nouvelles tiges annuelles, qui serait de 17136000 .

En divisant ensuite ce chiffre estimé par la quantité moyenne de tiges collectées annuellement par femme, on peut déduire le nombre de femmes pouvant y collecter «durablement » des tiges, c'est-à-dire sans dépasser le taux de renouvellement naturel des tiges.

Ainsi, avec un prélèvement estimé de 50000 tiges/ an/femme, la pression maximale d'exploitation du marais d'Amparahibe est évaluée à 347 femmes. Il s'agit de la pression à ne pas dépasser pour ne pas compromettre la régénération naturelle.

Pour confronter cette estimation avec une pression globale vraisemblable, il est nécessaire de connaître le nombre total de femmes venant collecter des tiges. Grâce au nombre d'habitants de la zone de Manombo (estimé à 9000 personnes; ANGAP, 2003), il est possible d'évaluer le nombre de ménages et donc le nombre minimum de femmes exploitant le marais d'Amparahibe. En admettant qu'un ménage compte six personnes et qu'il y a au mini- 
mum une vannière par ménage, le nombre de femmes suceptibles de collecter des tiges dans ce marais est au minimum 1 500. Ce nombre, représentant une estimation de la pression minimale d'exploitation réelle, se situe bien au-delà de la pression maximale estimée admissible sur le marais. Ce raisonnement rapide suggère la surexploitation du marais d'Amparahibe. Il pourrait s'appliquer à d'autres marais pour guider des décisions de gestion.

Au sein des trois zones, une forte dépendance économique de très nombreux ménages à l'égard de la vannerie a été constatée. Cette activité fait largement partie de l'organisation productive et permet de faire face à la faiblesse et à l'instabilité des revenus agricoles. Elle constitue souvent l'unique source de revenus et permet de ce fait de se fournir en produits de consommation de base. En plus de souligner le rôle primordial de la vannerie pour les ménages ruraux, l'étude socio-économique a permis de mettre en évidence un nombre non négligeable d'acteurs intervenant dans la filière régionale de la vannerie.

\section{Annexe 1. \\ Enquêtes vannières.}

\section{Paramètres généraux propres à la vannerie}

\section{Les étapes de la fabrication}

Pouvez-vous me nommer et me décrire toutes les étapes nécessaires à la confection de paniers ou de nattes ainsi que la durée de chacune d'elles?

Avez-vous d'autres frais entrant dans la confection des paniers (consommations intermédiaires comme les colorants, lanières en plastique...) ?

Quels types d'objets fabriquez-vous et à quel nombre par semaine ? Connaissez-vous la quantité de tiges dont vous avez besoin pour la confection d'un panier, d'une natte... ? Qui dans votre famille pratique la vannerie ? Y a-t-il répartition des tâches ? Si oui, comment s'organise cette répartition (par âge, par moment de l'année, par sexe...) ? Qui part vendre les paniers aux marchés?

Combien de temps consacrez-vous à la vannerie par jour? Quels sont les avantages que présente cette activité ? Les inconvénients?

\section{La vente des produits}

Combien en vendez-vous en moyenne par semaine ? Choix et quantité des objets fabriqués en fonction de quoi (de la demande, du temps disponible...) ?

Quel est le prix de vente des différents objets ? Les prix varient-ils durant l'année?

Y a-t-il un bon écoulement de la marchandise ? Devez-vous la stocker longtemps?
Néanmoins, l'étude a permis de relever que cette filière est sans doute responsable d'une surexploitation de l'espèce collectée. Face à cette situation, il est impératif de prendre des dispositions limitant ce phénomène en mettant en place une gestion des peuplements de mahampy. Particulièrement, des plantations de Lepironia mucronata, gérées durablement, permettraient à moyen et long terme de répondre aux besoins en tiges d'une partie des vannières tout en diminuant la pression sur les marais naturels. En effet, d'après une étude de RANDRIATAFIKA et al. (2007), de nouveaux peuplements de $L$. mucronata issus de plantations nécessitent entre 21 et 33 mois pour atteindre les mêmes valeurs biométriques que les peuplements naturels. Ces plantations pourraient être installées dans des zones inondées attenantes aux fokontany et gérées de manière collective ou privée, comme le font déjà un petit nombre de ménages.

Cette stratégie permettrait de réduire les incessantes pénétrations dans les deux aires protégées et donc le risque de les voir se dégrader continuellement.

Devez-vous payer des taxes sur la vente des paniers ou une participation pour pouvoir vendre sur le marché ?

À qui vendez-vous vos paniers ? Pouvez-vous me décrire les acheteurs (sont-ce des grossistes, des particuliers...) ?

Quel est le revenu mensuel apporté par cette activité ? Gardez-vous une partie de l'argent gagné ou le dépensezvous immédiatement sur le marché pour acheter des produits de consommation?

\section{Paramètres propres à la ressource}

Quelles sont les espèces que vous utilisez dans cette activité et comment les nommez-vous ?

Quand se fait la collecte des tiges ? En période de récolte du riz, continuez-vous de collecter et de tresser?

Allez-vous prélever dans différents endroits ? Si oui, pourquoi et dans combien de sites ? Quelle est la fréquence des changements ? Est-ce loin du village (temps de marche) ? Combien de temps dure une opération de collecte?

Quelles en sont les règles d'accès et d'extraction ? Y a-t-il des délimitations ethniques, claniques des rivières ? Y a-t-il des exclusions ethniques ou claniques?

Y a-t-il des moments de l'année où vous avez des problèmes de disponibilité en cette matière première ? Cela correspond-il à une saison particulière ? Comment faites-vous pour remédier à ce problème?

Qu'en est-il de la régularité de production de ces marais à plus long terme? Y a-t-il des années où il y a moins ou pas de production?

Est-ce qu'il existe dans le dina des lois réglementant les collectes?

Y a-t-il des contraintes législatives (permis d'exploitation)? 
Annexe 2.

\section{Enquêtes chefs de ménage.}

\section{Description socio-économique des ménages}

\section{Le ménage}

Composition de la famille (nombre de personnes, âge et sexe).

Niveau d'éducation des membres de la famille.

Organisation de la force de travail potentielle par activités économiques (pêche, agriculture, vannerie, autre) et ratio du nombre d'actifs agricoles.

Appartenance ethnique et structure sociale (lignages, segments de lignage dans les villages voisins, ancêtres communs, histoire du lignage).

De quoi est composé votre terroir et quelle est son étendue? Ce terroir est-il celui de vos ancêtres ou avez-vous dû quitter les terres de vos ancêtres ? Si oui, depuis quand et pour quelles raisons?

\section{L'habitation}

Caractérisation (type de matériel, type de toiture, durée d'utilisation, état, nombre de pièces).

Possédez-vous un grenier, une aire de séchage, un poulailler?

Facteurs de reconstruction (cyclone, inondation...) ?

Télévision ? Radio ? Moyens d'éclairage ? Moyens de transport?

Depuis quand votre famille est-elle installée sur ce terrain ? Qui en héritera?

Accès à un puits aménagé ou une citerne ? Quel est le temps moyen par jour consacré à la collecte en eau (en minutes) et à la collecte de bois mort?

\section{Les activités économiques}

\section{L'agriculture}

Taille et nombre de parcelles cultivables.

Types de cultures et leurs rendements.

Calendrier cultural (types de cultures, étapes culturales et nombre de personnes nécessaires pour chaque étape).

Calendrier alimentaire. Quels sont les aliments de base et la consommation journalière par personne? Combien de mois êtes-vous autosuffisant en ces aliments de base?

Votre production vivrière actuelle est-elle suffisante pour dégager un surplus que vous pouvez vendre ? Si oui, quelle est la part de la production agricole et fruitière vendue? Nombre et types d'animaux d'élevage : achat-vente-frais.

\section{Les autres sources de revenus}

De quelles autres façons êtes-vous monétarisé (diversification des sources de revenus : vente de bois de chauffe, de vanille, de girofle, de poivre, de produits de la pêche (lesquels ? langoustes, ailerons de requin, poissons...), cultures de rente, vannerie, travail salarié...) ? Quelle est la fréquence de ces activités?

Montants des revenus engendrés par ces activités économiques (prix de vente (au kg) aux marchés) et à qui vendezvous (collecteurs ou particuliers) ?

Calendrier des revenus (ex. : pendant la saison cyclonique, pas de revenus de la pêche mais beaucoup de vente d'ananas aux collecteurs des environs). Quels revenus sont constants d'une année à l'autre ? Y a-t-il des sources de revenus qui sont complètement absentes certaines années? Lesquelles?

\section{Les dépenses}

Quels sont les investissements agricoles (achat ou location d'outils, produits phytosanitaires, engrais, engagement de travailleurs saisonniers), la fréquence et le lieu de ces achats? Quelle est la durée de vie d'un outil ?

Avec l'ensemble des revenus du ménage, arrivez-vous facilement à couvrir les dépenses de votre ménage ? Arrivezvous à faire face à vos besoins fondamentaux ?

Niveau de dépenses moyen de santé par ménage et par an. Niveau de dépenses moyen de scolarité par ménage et par an.

Calendrier des dépenses courantes et non courantes.

\section{L'organisation foncière}

Possédez-vous les terrains que vous cultivez ?

Possédez-vous des terres autres que cultivables? Si oui, quels types (savanes, forêts...) ?

Quels sont les modes d'accès à la terre (héritage masculin uniquement, métayage, achat, location, défrichage et culture de terres non occupées...) ? Y a-t-il des opérations de sécurisation foncière ? Y a-t-il des contrats écrits, des titres de propriété, de l'immatriculation foncière (bornage, cadastre) ? Si oui, devez-vous payer des taxes foncières?

Les formations marécageuses appartiennent-elles à la communauté villageoise ? Quelles en sont les règles d'accès et les règles d'extraction? Y a-t-il des délimitations ethniques, claniques des rivières ? Y a-t-il des exclusions ethniques ou claniques? 
Annexe 3.

\section{Enquêtes commerçantes de vannerie.}

À combien de femmes achetez-vous la production ? À quelle fréquence?

Dans combien de marchés?

Où et à qui revendez-vous la production ?

À quel prix achetez-vous la marchandise et à quel prix la revendez-vous (pour chaque type de produit)?

Quels sont vos frais?

Y a-t-il des contraintes législatives, institutionnelles et logistiques à vos opérations?

\section{Références bibliographiques}

ANGAP, 2003. Plan de gestion de la conservation de la Réserve spéciale de Manombo. Antananarivo, Madagascar, Association nationale pour la gestion des aires protégées.

CADOTTE M., RAKOTONASOLO F., REZA L., LOVETT-DOUST J., 2002. Tree and shrub diversity and abundance in fragmented littoral forest of southeastern Madagascar. Biodiversity and Conservation,11 (8): 1417-1436.

CONSIGLIO T., SCHATZ G. E., MC PHERSON G., LOWRY P., RABENANTOANDRO J., ROGERS Z. S., RABEVOHITRA R., RABEHEVITRA D., 2006. Deforestation and plant diversity of Madagascar's littoral forests. Conservation Biology, 20 (6): 1799-1803.

GANZHORN J. U., LOWRY P. P., SCHATZ G. E., SOMMER S., 2001. The biodiversity of Madagascar: one of the world's hottest hotspots on its way out. Oryx, 35: 346-348.

GREEN G. M., SUSSMAN R. W., 1990. Deforestation history of the eastern rain forests of Madagascar from satellite images. Science, 248: 212-215.

IKUSIMA I., 1978. Primary production and population ecology of the aquatic sedge Lepironia articulata in a tropical swamp, Tasek Bera, Malaysia. Aquatic Botany, 4: 269-280.

KREMEN C., RAZAFIMAHATRATRA V., PHILIP GUILLERY R., RAKOTOMALALA J., WEISS A., RATSISOMPATRARIVO J.-S., 1999. Designing the Masoala National Park in Madagascar based on biological and socioeconomic data. Conservation Biology, 13 (5): 1055-1068.

LE ROY E., KARSENTY A., BERTRAND A., 1996. Maîtrise foncière et gestion forestière. In : Le Roy E., Karsenty A., Bertrand A. (éds.), La sécurisation foncière en Afrique, pour un gestion viable des ressources renouvelables.. Paris, France, Karthala, 103-116.
MYERS N., MITTERMEIER C. G., DE FONSECA G. A. B., KENT J., 2000. Biodiversity and hotspots for conservation priorities. Nature, 403: 853-858.

RANDRIATAFIKA F., RAMANAMANJATO J. B., VINCELETTE M., SOLOARIVELO S., RANDRIHASIPARA L., 2007. Monitoring of biometric and ecological parameters following restoration of a Lepironia mucronata (Family Cyperaceae) wetland in Mandena. In: Ganzhorn J. U., Goodman S. M., Vincelette M. (eds). Biodiversity, Ecology and Conservation of Littoral Ecosystems in Southeastern Madagascar, Tolagnaro (Fort Dauphin). Series editor Alfonso Alonso. Washington, DC, États-Unis, Smithsonian Institution, 355-362.

RAZANAJATOVO R., 2006. Caractérisation des marécages de prélèvement de Mahampy (Lepironia mucronata, Cyperaceae) du site de Mandena, Taolagnaro, dans le cadre d'un aménagement rationnel après exploitation minière. Mémoire de fin d'études, École supérieure des sciences agronomiques, Département des eaux et forêts, Université d'Antananarivo, Madagascar.

REZA L., RAKOTOARIVONY F., BIRKINSHAW C., RANDRIANASOLO A., 2005. Conservation communautaire de la forêt d'Agnalazaha. Description et évaluation du site, stratégie de conservation. Rapport non publié pour Cantonnement des Eaux et Forêts, Farafangana, et Missouri Botanical Garden.

VERMEULEN C., CARRIÈRE S., 2001. Stratégies de gestion des ressources naturelles fondées sur les maîtrises foncières coutumières. In : Delvingt $W$. (éd.). La forêt des hommes. Terroirs villageois en forêt tropicale africaine. Gembloux, Belgique, Les Presses agronomiques de Gembloux, 107-141.

URFER S., 2009. Travailleurs des pays pauvres. Revue Projet (Ceras-Recherche et action sociales), 309. 